\title{
Corregimiento de Pueblo Bello: hacia un sueño de prácticas ecosóficas
}

\author{
Village of Pueblo Bello: towards \\ a dream of ecosophic practices
}

Cómo citar este artículo en APA: García-Marín, E. y Builes, C. (2021). Corregimiento de Pueblo Bello: hacia un sueño de prácticas ecosóficas. Analecta Política, 11(20), 43-63. https://doi. org/10.18566/apolit. v11n20.a03

Fecha de recepción: 09.06.2020

Fecha de aceptación: 19.03.2021

\section{EULALIA GARCÍA-MARÍN}

Doctora en Filosofía Corporación Universitaria Americana - Medellín Integrante del grupo de investigación TES

Correo:mgarcia@americana.edu.co

\section{CARLOS BUILES}

Doctor en Español

Programa Ciencia Política Universidad de Antioquia Integrante grupo Estudios Internacionales de la Facultad de Derecho y Ciencia Política de la Universidad de Antioquia Correo electrónico: calberto.builes@udea.edu.co 


\section{Resumen}

Este artículo da cuenta de la complejidad de la relación naturaleza, sociedad y cultura en el corregimiento de Pueblo Bello, en el departamento de Antioquia (Colombia). Las rupturas en el tejido social ocasionadas por la violencia han desvanecido el encuentro de lo humano con su entorno. La comunidad del corregimiento de Pueblo Bello es un ejemplo de cómo los acontecimientos trágicos pueden ser una oportunidad para tejer nuevas relaciones desde y con la naturaleza.

Palabras clave: Pueblo Bello, ecosofía, foros ambientales, violencia, alternativas al desarrollo

\section{Abstract}

This article gives an account of the complexity of the relationship between nature, society and culture in the village of Pueblo Bello, in the department of Antioquia (Colombia). The ruptures in the social fabric caused by violence have vanished the encounter of the human with its environment. The community of the village of Pueblo Bello is an example of how tragic events can be an opportunity to forge new relationships from and with nature.

Keywords: Pueblo Bello, ecosophy, environmental forums, violence, alternatives to development. 
La naturaleza no es lugar para visitar, es el hogar.

Gary Snyder

\section{Introducción}

La Ley 1448 de 2011 ha sido un instrumento estatal para el comienzo de una memoria histórica integral que conlleve el restablecimiento de la verdad, la justicia y la no repetición en la sociedad colombiana. El aislamiento y el silenciamiento de comunidades y víctimas del conflicto colombiano a lo largo del siglo XX han provocado en el país una especie de patología individual y colectiva que ya otras sociedades y países han vivido en tiempos de guerra. La negación a nombrar lo innombrable (la guerra) ha provocado efectos psicosociales y la nación tardará décadas para restablecer de nuevo la confianza colectiva.

El oscuro panorama de la guerra que ha enrarecido el conjunto de las prácticas sociales y las configuraciones culturales en muchos territorios del país ha eclipsado los esfuerzos de líderes y pequeñas sociedades locales por superar de forma creativa y propia su cruda realidad. Los déficits de integración y justicia en Colombia, paradójicamente, se ven contrastados con prácticas de nuevas ciudadanías en territorios rurales o limítrofes con la urbanidad.

El proceso de investigación del que es fruto este artículo busca visibilizar los esfuerzos de la comunidad y los líderes del corregimiento Pueblo Bello, en el departamento de Antioquia (Colombia), en la construcción de su propio tejido social después de la violencia. Un esfuerzo aún en proceso que tiene como novedad la relación que tenemos con la naturaleza como fuente de un nuevo contrato social.

Este trabajo se enmarca en el campo de las ciencias políticas, pero en particular en los estudios de violencia y paz. La complejidad de los conceptos de conflicto, violencia y paz hace que las investigaciones tengan la necesidad de tomar algún punto de partida para no caer en generalizaciones o lugares comunes.

En esta investigación, se entiende que hay diversas concepciones del conflicto. Cabe recordar la taxonomía que ofrece Serrano (2001) sobre los distintos discursos de conflicto. Primero, se remite a Platón y al cristianismo para afirmar que en ellos el conflicto es el fruto del rompimiento del orden o paraíso y, en ese sentido, es algo negativo que debe ser superado para volver al orden original. El segundo discurso lo refiere al contractualismo que afirma que el conflicto es necesario, pero que debe 
ser neutralizado por el procedimentalismo del contrato jurídico y social del Estado de derecho. La tercera noción de conflicto más contemporánea se refiere a la concepción de conflicto como necesaria y afirmativa, esto es, no es importante neutralizar los conflictos, sino más bien potenciarlos desde un entramado de lo político en las diversas formas de las democracias plurales. De hecho, esta última noción la ha desarrollado ampliamente Mouffe (1999) en una propuesta muy pertinente para la complejidad de nuestros tiempos, consistente en actualizar la importancia de lo político como el lugar agónico donde los conflictos pueden convertirse en oportunidades; en el fondo de esta investigación, se encuentra este discurso sobre el conflicto: como oportunidad de retornar a lo político.

No se pueden omitir las investigaciones de Galtung (2003) sobre la violencia y la paz. Si los conflictos no pueden ser neutralizados o potenciados activamente, pueden volverse círculos viciosos de violencias. El aporte de Galtung a los estudios de violencia y paz consiste en hacer aparecer que la violencia no solo es un acontecimiento directo sobre el cuerpo individual o social, sino que es un entramado que responde a una violencia institucional y estructural, que, en no pocas ocasiones, se legitima con elementos de la cultura como la religión, la lengua, la ciencia o el arte.

\section{Metodología}

La investigación "Ecosofía: un nuevo en Pueblo Bello", presentada en su primera fase por el grupo de investigación Epimeleia de la Escuela de Teología, Filosofía y Humanidades y por el Grupo de Investigación en Estudios Políticos de la Facultad de Ciencias Políticas de la Universidad Pontificia Bolivariana, es un esfuerzo de trabajo cooperativo que tiene el propósito de hacer un estudio interdisciplinario que permita comprender las complejas conexiones entre el centro y los territorios, y toda su configuración cultural, social y natural.

Se ha tomado como lugar de investigación el corregimiento de Pueblo Bello, en el departamento de Antioquia (Colombia). Su elección no ha sido algo casual, sino que está enmarcada en los efectos "socioecológicos" de la intervención del Estado colombiano en la zona a través del llamado Contrato Plan Atrato Gran Darién ${ }^{1}$ que incluía al municipio de Turbo.

1 El Contrato Plan Atrato Gran Darién es un esfuerzo de política pública territorial para la paz en una de las zonas más quebradas por el conflicto armado. 
Se buscaba, en la primera fase de la investigación, identificar los efectos sociales de la pavimentación de la carretera El Tres (Turbo) hacia el municipio de San Pedro de Urabá, pero, al conocer las realidades socioambientales de la comunidad de Pueblo Bello (la cual se encuentra en medio de los municipios de Turbo y San Pedro de Urabá), nos dimos cuenta de que allí se estaban gestando nuevas formas de habitar lo rural que estimulaban tejidos sociales desde la relación con la naturaleza. Esa fue la razón por la que metodológicamente incorporamos a la investigación los conceptos de ecosofía de Guattari (2015) y de contrato natural de Serres (2004), pues pensábamos que podrían describir bien aquellas realidades ecosóficas que estábamos presenciando en el corregimiento de Pueblo Bello.

La exploración se hizo durante 2016 a través de la observación participante en conferencias y talleres a los cuales fuimos invitados los autores, así como de inmersiones en la comunidad con la que vivimos las realidades cotidianas. También se recopiló información a través de la observación no participante y se realizaron algunas entrevistas en profundidad al fundador del corregimiento de Pueblo Bello, el señor Saturnino Delgado, y a algunas de las lideresas de la comunidad. Tuvimos la oportunidad de hacer una entrevista colectiva a un grupo de ciudadanos que ayudaron a crear el centro Remanso de Paz, que equivaldría a la casa de la memoria del corregimiento de Pueblo Bello.

Presentamos, entonces, el contexto geográfico, histórico y social de la región del Urabá antioqueño para visibilizar la situación particular de violencia y resiliencia que ha vivido la comunidad del corregimiento de Pueblo Bello del municipio de Turbo, en el departamento de Antioquia. Se hace énfasis en los acontecimientos más violentos vividos en la década de 1990 para mostrar la manera en que sus habitantes han construido tejidos sociales y simbólicos a partir del entramado con la naturaleza.

\section{Las paradojas del Urabá colombiano}

\section{Ubicación geoestratégica y su proyección hacia el futuro}

La región del Urabá antioqueño ha representado, desde hace más de un siglo, el sueño de los antioqueños por llegar al mar (Caribe) y al Atrato (Pacífico). Este proyecto social que se ha convertido en un eje geoestratégico desde el Gobierno y el mundo empresarial antioqueño ha sido vertical (una nueva forma de colonialis- 
mo) y no ha considerado las realidades y aspiraciones propias de las comunidades del Urabá. Actualmente, el Gobierno departamental está diseñando tres grandes estrategias de intervención en Urabá: los puertos, ${ }^{2}$ la interconexión vial-social ${ }^{3}$ y la restitución simbólica, en el posconflicto, o posacuerdo, como se prefiere llamar en el mundo académico.

El departamento de Antioquia cuenta con una gran riqueza cultural, social y ambiental debido a la diversidad de las regiones que lo componen: la región metropolitana que abarca a Medellín y nueve municipios más, la región del oriente antioqueño, la del norte, la del nordeste, la del Bajo Cauca, la del suroeste, la del occidente y la de Urabá que comprende territorios tanto de cultura del Pacífico chocoano (Vigía del Fuerte y Murindó) como de cultura caribeña (Chigorodó, Carepa, Apartadó, Necoclí, San Juan de Urabá y Arboletes). Existen, además, dos municipios de frontera que comunican, el uno con el occidente antioqueño, Mutatá, y el otro comunica con el departamento de Córdoba, el municipio de San Pedro de Urabá. El corregimiento de Pueblo Bello, objeto de estudio de esta investigación, se encuentra en el municipio de Turbo, pero colinda con el municipio de San Pedro de Urabá. El corregimiento de Pueblo Bello se encuentra situado al borde de la carretera que conduce de El Tres (Turbo) hacia el municipio de San Pedro de Urabá (figura 1).

2 En el Urabá antioqueño, se planea la construcción de tres puertos: Puerto Pisisi ubicado en la vereda Casanova (municipio de Turbo) según la Resolución 297 de 2017 de la Autoridad Nacional de Licencias Ambientales (ANLA); Darién International Port (Puerto Darién-Necoclí) según la Resolución 1092 de 2017 de la ANLA, y Puerto Antioquia ubicado en el corregimiento de Nueva Colonia (municipio de Turbo) según Resolución 78 de 2016 de la ANLA.

3 Está en construcción el proyecto de las Autopistas para la Prosperidad en el departamento de Antioquia, el tramo autopista Mar 1 con intervenciones desde el municipio de Cañasgordas hasta el corregimiento de El Tigre en el municipio de Chigorodó, y Mar 2 entre los municipios de Chigorodó y Necoclí. También se encuentra en construcción el túnel del Toyo entre los municipios de Giraldo y Cañasgordas que mejorará la interconexión vial entre Medellín y Urabá. El Gobierno Nacional también ha intervenido a través del Contrato Plan Atrato - Gran Darién las carreteras anexas al Atrato (Caucheras-Belén de Bajirá-Riosucio) y la interconexión vial que comunica la municipio de Turbo con la ciudad Córdoba desde el corregimiento de El Tres, pasando por el corregimiento de Pueblo Bello hasta el municipio de San Pedro de Urabá (Resolución de 2016 de ANLA). 
Figura 1. Mapa de la región del Urabá.

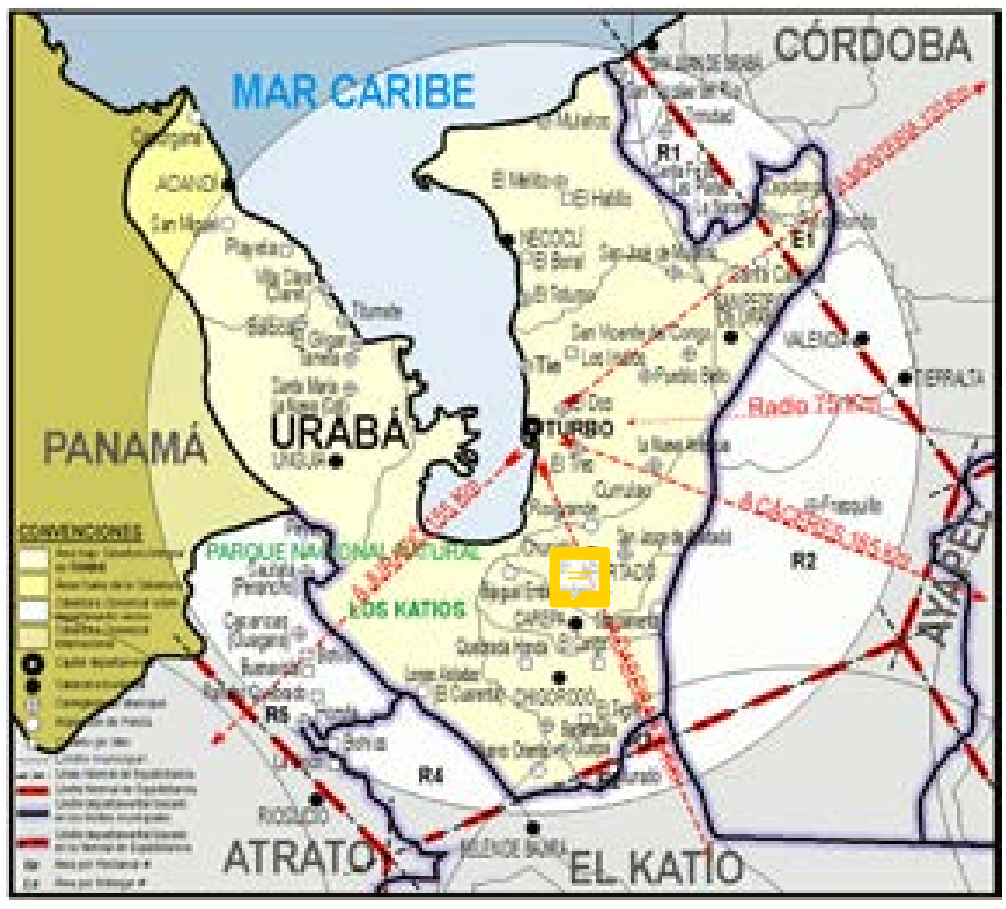

Fuente: Logistica424 (s. f.).

El Urabá antioqueño tiene una extensión de $11664 \mathrm{~km}^{2}$, lo que equivale al 18,6 \% de la extensión del departamento de Antioquia, valga decir, es la región más extensa del departamento. Según el Departamento Administrativo Nacional de Estadística (DANE), citado en Cámara de Comercio de Urabá (2016), Urabá cuenta con 706000 habitantes de los cuales el $49 \%$ son mujeres y el $51 \%$ son hombres. Sobresalen en el campo económico y productivo los cultivos de banano y, en menor escala, los de plátano y cacao, así como la ganadería extensiva. Sin embargo, la potencialidad del territorio va más allá de la producción local. Desde el mundo empresarial, nacional e internacional, esta región es considerada como geoestratégica, por lo que se han propuesto diferentes instrumentos de planeación e intervención. 


\section{La planeación centralizada hacia los territorios: Antioquia la mejor esquina}

Sarmiento (2007) propone una nueva interpretación en los estudios críticos sobre Urabá. Su mirada antropológica y sociológica ilustra de forma transversal el complejo rizoma que esconde el aparatoso plan empresarial de control territorial sobre una zona que no cesa de buscar su propia identidad y de estar en construcción. La lucha por sus riquezas y su ubicación geoestratégica ha ido produciendo migraciones diversas con intereses y posturas ideológicas, económicas y militares que en muchas ocasiones han convertido este territorio en un experimento de guerra.

La historia del conflicto colombiano pasa con todos sus ejes por Urabá: el conflicto por tierras y monocultivos, los desplazamientos de la Violencia de la década de 1950, la configuración de las guerrillas y su posterior desplazamiento por el paramilitarismo y el enfoque militarista de Estado, la llegada de desplazados de los departamentos de Córdoba y Chocó, y el neocolonialismo del empresariado antioqueño a la región, a través de su plan estratégico para 2020 "Antioquia la mejor esquina de América, justa, pacífica, educada, pujante y en armonía con la naturaleza" (Visión Antioquia Siglo XXI). Este último plan buscaba convertir al departamento de Antioquia, y por supuesto a Urabá, en el centro geoestratégico de América. La cuestión problema consistía en que ese ejercicio de planeación era una decisión unilateral economicista que buscaba desarrollar los principales negocios y empresas departamentales sin considerar la compleja realidad nacional y los intereses y proyectos estratégicos de las comunidades locales.

\section{La violencia y la pobreza multidimensional en el Atrato y Gran Darién}

En contraste con la idea utópica y de carácter colonialista de Antioquia Visión Siglo XXI, aparece la realidad de pobreza multidimensional de Urabá donde la brecha social es tan grande que en su conjunto el Atrato y el Gran Darién es una de las regiones con menor desarrollo humano del país. Entiéndase la paradoja de los discursos de desarrollo que esconden según su intencionalidad un modelo de progreso económico industrial o aquel que desenmascara ese discurso dominante y presenta alternativas al "desarrollo" desde el diálogo mismo con nuestras culturas ancestrales en América Latina (Escobar, 2016, 2017, 2018, 2019; Noguera, 2004, 2007, 2010, 2014, 2016, 2018, 2020). 
Según el Acuerdo Estratégico Contrato Plan Atrato - Gran Darién:

La región Atrato Gran Darién presenta hoy uno de los peores índices de desarrollo y pobreza del país. Su población es la más expuesta al conflicto y a la violencia, a la marginalidad y a la explotación indiscriminada de su territorio y de sus recursos naturales, y presenta altas tasas de mortalidad infantil, pobreza y pobreza extrema, precariedad en el acceso y calidad de los servicios públicos vitales para el bienestar y segmentación económica y social.

Este contraste entre una región con gran presencia empresarial nacional e internacional y unas condiciones de desarrollo humano tan bajos potencializa el conflicto social al máximo y crea condiciones para que los actores ilegales asuman el relevo estatal en materia de seguridad, además, el control de la vida individual y social; tal es el caso del corregimiento de Pueblo Bello.

\section{Corregimiento de Pueblo Bello: búsqueda de nuevos horizontes de paz}

\section{Un encuentro cultural entre los departamentos de Antioquia y Córdoba}

El corregimiento de Pueblo Bello se encuentra ubicado en medio de los municipios de Turbo y San Pedro de Urabá en el departamento de Antioquia. Es un poblado aproximadamente de 4000 personas que se encuentran en el casco urbano y rural. Tiene ocho veredas: Sinaí, Monomacho, La Ilusión, El Caucho, Lucio, Galilea, La Esperanza y La Unión, nombres que expresan el anhelo de sus habitantes de vivir en paz.

Sus pobladores originales llegaron del departamento de Córdoba hacia la década de 1950 en busca de tierra para colonizar. En aquel entonces, según nos cuenta uno de los primeros fundadores del pueblo, el señor Saturnino Delgado, "ya estaban en territorio, explorando posibilidades, las compañías americanas farmacéuticas que buscaban comercializar raíces de plantas y árboles endémicos para el uso químico". En ese encuentro entre los primeros colonizadores que buscaban conquistar y dominar la naturaleza salvaje, se sitúan los orígenes del corregimiento de Pueblo Bello. Más tarde llegaron pobladores antioqueños que fueron comprando tierras ya abiertas por cordobeses y se arraigaron en el territorio. Tres inculturas 
(Tunubalá 2020) y cosmovisiones aparecen en el corregimiento de Pueblo Bello: la del cordobés que es la figura humana y cultural que prevalece hasta el día de hoy; la del antioqueño que se ubicó en su mayoría en un corregimiento cercano, Alto de Mulatos, y la del norteamericano que avizoró en aquellas tierras potenciales madereros (maderas rojas) y raicillas para uso químico. Del paso de los norteamericanos por la región quedó una huella religiosa que hasta hoy es perdurable: la religión protestante (evangélicos de diferentes nominaciones).

La fundación del corregimiento de Pueblo Bello fue materializada con la creación de la primera escuela, que estaba ubicada donde hoy es el colegio. Una interesante característica de esta comunidad es que el colegio se ha constituido en su centro académico y cultural. ${ }^{4} \mathrm{La}$ actividad económica de los habitantes ha sido la ganadería y la agricultura, en especial, el cultivo del plátano, la yuca, el maíz y el arroz. Es importante anotar que el corregimiento de Pueblo Bello se encuentra en el borde de la carretera entre El Tres y el municipio de San Pedro de Urabá, ${ }^{5}$ lo que implica problemas de integración comunitaria, pero, paradójicamente, abre posibilidades de comunicación interdepartamental.

\section{Conflictos, violencias y construcción de paz territorial}

El círculo vicioso de la violencia ${ }^{6}$ que se tomó a Urabá a partir de la década de 1980 determinó el devenir histórico de esta región. La histórica presencia de movimientos liberales que habían llegado a la zona desde la Violencia y la instauración del control territorial por parte de las guerrillas propiciaron unas condiciones históricas que contrarrestaban el plan estratégico empresarial y estatal de retoma de la región. El nudo del círculo vicioso de la violencia se desató cuando grupos de autodefensas campesinas de Córdoba (llamados también paramilitares) comenzaron una guerra de control territorial contra las guerrillas y se desató una violencia multifacética, que tiene consecuencias hasta el día de hoy. Masacres,

4 El colegio de Pueblo Bello es el centro académico y cultural. Se encuentra cerca al centro de memoria histórica Remanso de Paz.

5 La carretera entre el corregimiento de El Tres, que es la intersección de la carretera al mar hacia el municipio de San Pedro de Urabá, ha sido pavimentada por medio de la intervención estatal Ilamada Contrato Plan Atrato - Gran Darién (2012-2018). Esta carretera fue el camino ancestral de los fundadores y se había convertido en un dolor de cabeza para los pobladores por su pésimo estado.

6 Término utilizado por Galtung (1989) para describir el movimiento perverso que genera la violencia directa, estructural y cultural cuando los conflictos no pueden ser mediados a través de la palabra o la ley. 
desapariciones forzosas, desplazamiento de campesinos, estigmatización de comunidades, etc., fueron los efectos en la población civil de aquella batalla territorial. El corregimiento de Pueblo Bello se encontraba estratégicamente ubicado para garantizar ese control; por eso, su comunidad, caracterizada por la buena organización social y el trabajo colectivo, fue estigmatizada por las autodefensas como protectora y simpatizante de la guerrilla, pero, contradictoriamente, la guerrilla vio con sospecha a algunos de sus habitantes como informantes de las autodefensas.

Tanto la guerrilla como los grupos de autodefensa ocasionaron desplazamientos y masacres en el corregimiento de Pueblo Bello, no sin el silencio o la complicidad misma del Ejército. La masacre más difundida por los medios de comunicación la ocasionó el grupo paramilitar Los Tangueros bajo el mando del conocido jefe Fidel Castańo. La historia narra que el 14 de enero de 1990 entraron en el caserío 60 paramilitares armados y con lista en mano empezaron a sacar a pobladores hombres de sus casas y los condujeron a la finca Santa Mónica y posteriormente a la finca Las Tangas del departamento de Córdoba. Fueron 43 hombres asesinados con sevicia, como lo narra la Corte Interamericana de Derechos Humanos: "durante dichos interrogatorios, a algunos de los secuestrados les cortaron las venas, las orejas, los órganos genitales o les chuzaron los ojos" (Corte Interamericana de Derechos Humanos. Caso de la masacre de Pueblo Bello vs. Colombia. Sentencia de 31 de enero de 2006). Tales perversiones, ya antes vistas en la Violencia (1946-1957), tuvieron como causa el robo de 43 cabezas de ganado por grupos de guerrilleros, quienes, en el mes de diciembre anterior a la masacre, transportaron los animales hacia el corregimiento de Pueblo Bello.

La masacre de los 43 habitantes hombres se ha convertido en un signo trágico que acompaña los relatos de la memoria histórica del conflicto armado colombiano, dado que esta pudo haber sido evitada si las autoridades, en especial el Ejército, hubieran tomado medidas preventivas. Ante la gravedad de la omisión intencional por parte del Ejército, que conoció del camión con paramilitares que se dirigía hacia el corregimiento de Pueblo Bello a realizar la masacre, el Estado colombiano fue condenado por la Corte Interamericana de Derechos Humanos en 2006 a la restitución integral de la comunidad de este corregimiento. 


\section{La restitución física y simbólica: una obligación internacional impuesta al Estado colombiano}

La acumulación de violencias en el corregimiento de Pueblo Bello lo convirtió en un pueblo fantasma, puesto que muchos de sus habitantes huyeron de este. Años más tarde, gracias a la obligación del Estado de restituir material y simbólicamente a sus habitantes, este comenzó de nuevo a repoblarse, no solo con sus antiguos habitantes, sino con otras personas desplazadas de la zona, quienes encontraron en el corregimiento abrigo y nuevo hogar. Desde 2012, el Estado colombiano, a través de la Unidad para las Víctimas, constituida jurídicamente por la Ley 1448 de 2011, ha comenzado una presencia más integral con el deseo de posibilitar las condiciones adecuadas para la conformación de la restitución del tejido social perdido.

$\mathrm{Al}$ mismo tiempo, las mujeres, los líderes sociales y los grupos religiosos presentes en el corregimiento de Pueblo Bello pensaron en idear maneras de cambiar el orden establecido; así, aparecen las propuestas de creación de grupos ambientales, cooperativas y fundaciones que buscaban mejorar las condiciones de vida de todos los habitantes en los diferentes aspectos. Esta iniciativa será el comienzo del tejido de una red en que lo femenino y el sentido ecológico se encuentran para dar como resultado diversas propuestas, entre ellas, los diferentes foros ambientales ecopaz, que fueron el nódulo de la observación-participación de los autores de este artículo. ${ }^{7}$

7 En 2016, los autores de este artículo participamos como ponentes en los dos foros ambientales Ecopaz que se realizaron en el 15 de abril y el 11 noviembre del mismo año. Además, tuvimos la oportunidad de realizar talleres con líderes, realizar una entrevista al fundador del pueblo y hacer un coloquio con los integrantes del museo de la memoria Remanso de Paz. Esta fue, también, la ocasión de convivir con los habitantes en su realidad cotidiana. Además, seguimos la ruta que recorrieron los 43 habitantes que fueron masacrados por los paramilitares. 


\section{Los foros ambientales ecopaz en el corregimiento de Pueblo Bello: una práctica ecosófica}

Introducimos el concepto de ecosofia ${ }^{8}$ en la vivencia de la comunidad del corregimiento de Pueblo Bello, porque desde esta noción es posible la comprensión “de las realidades complejas del mundo contemporáneo" (Guattari, 2015, p. 13), toda vez que nos permite integrar los aspectos teóricos y prácticos desde la abstracción filosófica. Asimismo, acudimos a este concepto para apoyarnos en la propuesta ecosófica como núcleo que puede permitir la creación de una nueva sociedad a partir de prácticas integradoras y la construcción de tejido social, de forma tal que se propicie una cultura del humanismo en que se restablezcan lazos en esta comunidad que permitan trascender todo ese padecimiento vivido después de los hechos violentos sucedidos en su territorio entre 1990 y 2000, que los llevaron a salir de su "espacio doméstico" (p. 29), porque fueron obligados a dejar sus lugares queridos, tras lo cual se dio una ruptura, un quiebre con el territorio, con el afecto y con las redes invisibles construidas con el lugar habitado.

En este contexto, nos apoyaremos en la propuesta ecosófica planteada, porque nos permitirá interpretar los foros ambientales ecopaz, realizados el 15 de abril y el 11 de noviembre de 2016, como una estrategia para relacionar la filosofía y la ecología, y así buscar integrar los diferentes aspectos de la sociedad para crear una nueva posibilidad de vivir en la mentalidad de la unidad y no en la de la fragmentación, y lograr nuevos propósitos que redunden en una sociedad más humana a partir de unas prácticas ecosóficas.

8 Planteamos la propuesta ecosófica de Guattari (2015), porque este concepto nos permite comprender en las vivencias observadas en la comunidad del corregimiento de Pueblo Bello cómo sus habitantes parten de ciertas creaciones contundentes como la fundación de la Cooperativa de Productores Agropecuarios Solución Integración y Vida (Proasiv), para construir e integrar otros aspectos. Por ejemplo, con la creación de Proasiv, ellos logran soberanía alimentaria y, a la vez, inician la creación de otras fundaciones y asociaciones que responden a otras necesidades de la comunidad en lo ambiental, cultural y educativo. Esta sinergia les permite crear todo un entramado de tejido social que se nutre, en gran parte, de la naturaleza. 


\section{Primer Foro Ambiental Ecopaz, 15 de abril de 2016}

El Foro Ambiental Ecopaz estuvo dirigido a la zona norte del municipio de Turbo: San José de Mulatos, San Vicente del Congo, Nueva Granada, Alto de Mulatos, El Tres y Pueblo Bello. Los organizadores se plantearon como objetivo

Promover la construcción de paz en la zona norte del municipio de Turbo, mediante la concientización de la responsabilidad ambiental, de manera individual, familiar y colectiva, del sector rural como expresión de superación del conflicto y fortalecimiento del trabajo en equipo de la comunidad, del sector público y privado, que aporte a la protección del medio ambiente para alcanzar un verdadero desarrollo sostenible. (Archivo de la Fundación Arrieros por la Paz)

Con este objetivo, se reafirma la intención que tienen los organizadores del Foro Ambiental Ecopaz de articular todos los distintos aspectos de la sociedad para cuidar los recursos naturales: agua, aire, suelo, flora y fauna, así como la vida humana, lo que evidencia una preocupación por el cuidado de los ecosistemas, con el objetivo de reflexionar frente a las condiciones de la zona norte en un asunto común que llama e invita a todos los de la comunidad (que tienen diferentes formas y grados de conocimiento) para propiciar la retroalimentación de la problema y conservar en óptimas condiciones el territorio.

Los habitantes del corregimiento de Pueblo Bello, de sus veredas y de los municipios cercanos asistieron masivamente al Foro Ambiental Ecopaz, muchos de ellos jóvenes y campesinos, los cuales participaron en las diferentes actividades académicas, pedagógicas y lúdico-ambientales en los distintos espacios comunitarios; asistieron más de 700 participantes.

Asimismo, los ejercicios teórico-prácticos para jóvenes y adultos sobre el manejo de residuos sólidos con la elaboración previa, por parte del comité ambiental de estudiantes de la Institución Educativa de Pueblo Bello, de canecas con botellas de plástico PET para recoger residuos, bolsas para mercar hechas con las reatas con que se amarran las cajas de banano y diferentes souvenirs elaborados con materiales reutilizables. Toda una propuesta de trabajo comunitario con fines ecológicos y educativos en reciprocidad con la naturaleza.

Se realizó también la jornada de siembra masiva, por parte de la comunidad de los estudiantes y los asistentes al foro, de mil plantas nativas protectoras en la ribera del río Mulatos y en lugares previamente organizados para ello. Este río es un signo histórico que narra el conflicto armado y el deterioro de la naturaleza. 
La lucha contra su abandono y contaminación se ha convertido en una nueva forma de crear conciencia y tejido social en una comunidad en construcción que pone en el centro de la integración de los registros ecosóficos (Guattari, 2015) la conservación de la naturaleza (figura 2).

Figura 2. Campaña de reforestación de las riberas del río Mulatos en el corregimiento de Pueblo Bello en la preparación del primer Foro Ambiental Ecopaz.

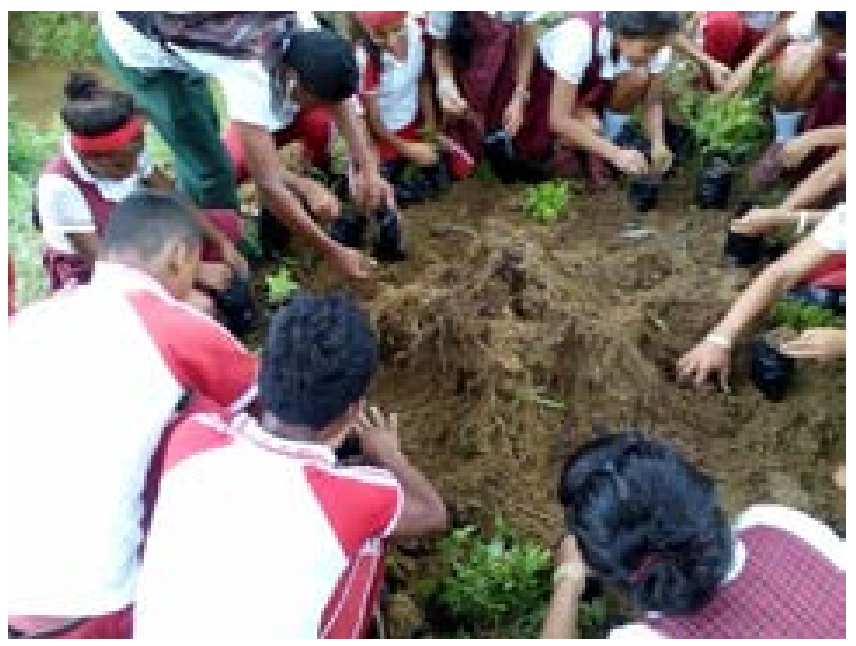

Fuente: Fotografía de Yesenia Luna Oviedo.

También se expusieron las maquetas ambientales que representaban el deterioro de los ecosistemas y las estrategias para su conservación. Cada situación estaba ilustrada con ejercicios prácticos que realizaban los asistentes, en que se pudieron contrastar las dos situaciones: ecosistemas deforestados y ecosistemas reforestados, y lo que ello implica para la naturaleza. Paralelamente, los expositores invitados presentaron las conferencias sobre los siguientes temas: variabilidad climática, deforestación, malas prácticas agrícolas, malas prácticas ambientales y acuíferos.

Al final del primer Foro Ambiental Ecopaz, se realizó la firma de pactos y responsabilidades compartidas para el segundo foro a realizarse en noviembre de ese mismo año. En la firma de los pactos, se integraron los representantes de las organizaciones del Estado, Policía y Ejército, así como Corporación para el Desarrollo Sostenible del Urabá (Corpourabá), el Programa de las Naciones Unidas para el Desarrollo (PNUD), la Corporación Ambiental de Pueblo Bello (Corampube), la Fundación Arrieros por la Paz, la Junta de Acción Comunal, la Cooperativa de 
Productores Agropecuarios Solución Integración y Vida (Proasiv) y la Secretaría de Educación y Salud del municipio de Turbo.

De la observación participante, pudimos tener un conocimiento más profundo no solo de la organización y del trabajo colectivo que caracterizan al corregimiento de Pueblo Bello, sino también de las costumbres y prácticas sociales en relación con las instituciones estatales. Exploramos un hilo tenue de desconfianza entre los grupos sociales que se organizaban por gobernanza con la administración local, en especial, con la alcaldía del municipio de Turbo.

\section{Segundo Foro Ambiental Ecopaz, 11 de noviembre de 2016}

En esta ocasión, el objetivo propuesto por los organizadores fue

promover la construcción de paz en la zona norte del municipio de Turbo, mediante la concientización de la responsabilidad ambiental de manera individual, familiar y colectiva del sector rural, como una expresión de superación del conflicto y fortalecimiento del trabajo en equipo entre la comunidad, sector público y privado que aportan a la protección del medio ambiente y alcanzar un verdadero desarrollo sostenible. (Archivo de la Fundación Arrieros por la Paz)

Se realizaron varias conferencias con un hilo conductor que enfatizó "la protección, conservación y preservación de los ecosistemas existentes en el territorio con el fin de construir paz desde la ruralidad denominada Ecopaz" (Archivo Fundación Arrieros por la Paz). Los temas presentados por diversos conferencistas fueron agua como política pública para el desarrollo rural integral, reforestación, agroecología, agricultura orgánica y seguridad alimentaria, manejo integral de los residuos sólidos, conservación de los ecosistemas y certificación en buenas prácticas agrícolas. Al mismo tiempo, se propuso un trabajo con los niños en unos espacios determinados para ello que se llamaron "estaciones" que fueron coordinadas por personas que tenían a su cargo los materiales y la implementación de la metodología específica para trabajar de manera lúdica los siguientes temas: manualidades navideñas reciclables, reforestación, manejo integral de los residuos sólidos, test ambiental y juegos lúdicos. Todo ello responde a la propuesta ecosófica de reinventar prácticas sociales que permitan un sentido de nuevas responsabilidades, lo que permite construir una sensibilidad tan profunda que conlleve la sabiduría para cuidar el hábitat (Guattari, 2015). 
También, y por iniciativa de grupos de mujeres de la población, se organizaron los refrigerios y almuerzos para ser entregados el día de la realización del foro. Con anterioridad, se convocó a las personas del comercio para que aportaran los alimentos con los que se prepararon los almuerzos. En la observación, se notó una articulación entre el sector comercial del corregimiento y las organizaciones sociales y ecológicas. Se crearon, entonces, equipos de cuatro mujeres que prepararon doscientos almuerzos y luego otro grupo de personas se encargaba de entregarlos a los asistentes al evento. Toda una red socioeconómica que nos permitió constatar ejercicios de prácticas de economía circular (Bajo, 2017). Esta colaboración entre todos los integrantes evidencia ese anhelo de integración que se propone la comunidad para construir una sociedad diferente: "para construir desde la teoría del caos nuevas formas de vivir, de sentir, de pensar [...] las inmensas transformaciones" (Guattari, 2015, p. 45). La propuesta de los foros ambientales ecopaz se perfila, entonces, como una alternativa diferente de llegar a realizar acuerdos locales que permitan trascender algunas condiciones históricas en que se han destruido o debilitado los tejidos sociales con gran cantidad de vejámenes que nos alejan del concepto de humanidad y que se traducen, en algunos casos, en sociedades angustiadas, temerosas, en que impera el caos que dificulta la creación del nuevo tejido social.

Por tanto, en los encuentros de los foros ambientales Ecopaz, se propicia la construcción de nuevos tejidos sociales al realizar una puesta en escena que integra los conocimientos teóricos y prácticos en torno a un problema común para los habitantes, en que cada uno aporta y participa desde su realidad. De esta forma, se integran deporte, arte, ambiente y música, en un entorno ecológico sostenible.

Lo que nos muestra la comunidad del corregimiento de Pueblo Bello es que, como el ave fénix, ellos también pueden resurgir de las cenizas, y crear relaciones de mutualismo, es decir, de ayuda, de confianza, de perdón y de reconciliación, desde las cuales se pueden replicar la reciprocidad de la naturaleza en la sociedad. En la medida en que los lazos con los otros seres humanos se afiancen a partir de nuevos acuerdos, colaboraciones y construcción de propuestas, se edifican otras relaciones comunitarias y se crean correlatos para generar nuevas perspectivas de vida. Esta propuesta parece un tanto compleja, máxime si consideramos que vivimos en una sociedad capitalista en que se han aplicado modelos de desarrollo economicista que perpetúan condiciones de inequidad al seguir, en algunos casos, las indicaciones internacionales de una economía de mercado.

A pesar de este marco económico-político global, la comunidad del corregimiento de Pueblo Bello intenta salir adelante con alternativas de desarrollo dis- 
tintas que se erigen desde la sociedad civil; evocamos la definición de Bauman (2017) sobre el concepto de utopia: "es ante todo una imagen de otro universo diferente al que se conoce por experiencia directa o por haber oído hablar de él" (p. 138). Es pertinente este llamado a la utopía porque las condiciones de sociedades afectadas por múltiples manifestaciones de violencia se repiten a lo largo de las últimas décadas y pareciera que nos acostumbramos y que estamos legando a nuestros descendientes valores, comportamientos y actitudes que dan cuenta de una cultura que perpetúa el círculo vicioso de la violencia.

Las prácticas ecosóficas, ${ }^{9}$ como las que proponen los habitantes del corregimiento de Pueblo Bello con los foros ambientales ecopaz, son una forma de modificar este círculo de violencia en tanto proponen recorrer el camino de lo impensable (lo utópico) para mostrar que es posible transformar las relaciones con los humanos y con el mundo, aprendiendo y adaptando nuevas sensibilidades en relación con la naturaleza. Esta nueva mentalidad de lograr lo imposible (la simbiosis naturaleza-sociedad) abre el camino hacia la construcción de ciudadanos soñadores de lo imposible.

Es necesaria esta esperanza en el presente, porque "los pueblos y los Estados no han encontrado hasta la fecha ninguna razón poderosa ni concreta para asociarse, para instaurar entre ellos una tregua larga" (Serres, 2004, p. 47). Es decir, se ha perpetuado un modelo de vivir con los otros, en algunos casos, permeado por circunstancias violentas que nos hace difícil convivir en sociedad. Una manera de romper con estas dificultades, luego de los episodios violentos en la región objeto de estudio, es propiciar nuevos encuentros, en este caso, los foros ambientales ecopaz, que aportan a un nuevo modelo de desarrollo proponiendo una transversalidad de todas las manifestaciones de la vida en comunidad.

La experiencia de los foros se presenta como un ejemplo de la construcción de la ecología política a partir de la integración de diferentes registros ecosóficos como lo ambiental, lo económico, lo social y lo mental, que buscan crear nuevos espacios; en palabras de Guattari (2015), espacios "cotidianos, personales, familiares, de vecindades" (p. 36), que propicien crear lazos fraternos, renueven las prácticas sociales habituales y generen una sabiduría profunda.

9 Prácticas ecosóficas derivadas de la integración entre los distintos aspectos de la comunidad, que generan unas relaciones simbióticas de mutualismo, comensalismo y reciprocidad, las cuales se traducen en la ayuda, la colaboración y la vivencia que hacen los niños, los jóvenes, los adultos, las familias y los comerciantes, desde sus distintos lugares existenciales de apropiación y aporte a la vecindad. 
De esta forma, se busca lograr un modelo diferente de desarrollo rural integral que incluya los aspectos cualitativos, y esto es posible si logramos una mirada profunda de los problemas vitales como la vida, la relación con los otros, la sensibilidad hacia los otros seres vivos y la construcción de un ecosistema social que le permita a la comunidad construir un mejor futuro para sus descendientes. Con la propuesta de desarrollo integrador, se pretende reorientar los medios y las finalidades de la producción para conservar la biósfera, la vida humana, animal y vegetal, propiciando el trabajo colaborativo para el cuidado del entorno en el momento presente, pero con una perspectiva del porvenir.

\section{Conclusiones}

Encontramos en el corregimiento de Pueblo Bello el ejemplo de una comunidad que, pese a una historia común atravesada por diferentes manifestaciones de violencia, está creando diversas formas de salir de ese pasado a partir de unas iniciativas y desde diferentes perspectivas. Una muestra de ello son los foros ambientales ecopaz en que es posible constatar tales iniciativas para un modelo de desarrollo alternativo. En medio de las dificultades, estos encuentros se han ido constituyendo en una práctica de resiliencia como "un nuevo tipo de desarrollo cualitativo que rehabilite las singularidades" (Guattari, 2015, p. 31). Hay que reconocer que, aunque se ha elegido la propuesta de Guattari como guía, desde América Latina autores como Arturo Escobar han presentado sendos trabajos que deben ser también considerados en futuras investigaciones.

Estos foros han sido posibles porque entretejieron las diferencias y las singularidades en medio de los disensos, y lograron establecer acuerdos socioambientales para propiciar una nueva mirada, una manera diferente de hacer las cosas, que dio como resultado la propuesta de una comunidad modélica. En esta propuesta de trabajo colaborativo, se evidenció el eje transversal de las distintas actividades realizadas por diferentes actores de la sociedad, para gestar una nueva manera de vivir en que se superen las dificultades del pasado y se pueda generar memoria a partir de creaciones colectivas diferentes. Tal colaboración también da cuenta de ese anhelo de integración que se propone la comunidad para construir soluciones específicas que ayuden a crear un mejor porvenir, al orientar sus inquietudes en los encuentros de los foros ambientales ecopaz a reflexionar sobre los temas que los interrogan y que se irradian hacia otras poblaciones del territorio y que están sembrando la semilla de la paz. Esta experiencia investigativa nos permitió constatar que, aun en medio de las condiciones más adversas en que las comunidades 
pueden vivir situaciones de violencia, es posible, desde la cooperación y la reciprocidad, transformar las realidades que se viven y el entorno.

Finalmente, se puede colegir que los foros ambientales ecopaz pueden constituirse en un instrumento de encuentro y conciliación, porque, al proponer la participación de los diferentes integrantes de la población, se logra que las distintas visiones e interpretaciones de los problemas se integren en una propuesta ecosófica. Este trabajo, construido entre los diferentes grupos, movimientos sociales y organizadores, está logrando tejer un clima de paz y concordia desde los distintos registros ecosóficos como lo social, lo ambiental, lo mental y lo ético-político (figura 3).

Figura 3. Imagen del Segundo Foro Ambiental Ecopaz en que participaron PNUD, Prosperidad Social, Plan Nacional de Desarrollo 2014-2018 "Todos por un nuevo país", Ministerio de Agricultura, Alcaldía de San Pedro de Urabá, Fundación Arrieros por la Paz, Corampube, Proasiv y Universidad Pontificia Bolivariana

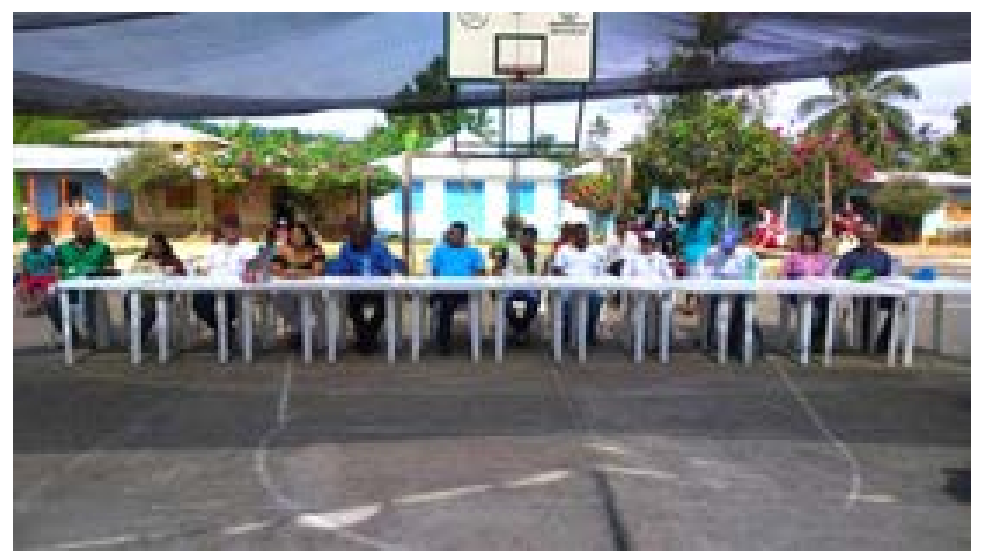

Fuente: Fotografía de Yesenia Luna Oviedo.

\section{Referencias}

Acuerdo Estratégico Contrato Plan Atrato - Gran Darién. https://pactosterritoriales.dnp. gov.co/contratosplan/contrato-plan-atrato-gran-dar\%C3\%ADen

Autoridad Nacional de Licencias Ambientales. (2016, 28 de enero). Resolución 78. Por la cual se modifica la licencia ambiental otorgada mediante la resolución $n^{\circ} 0032 \mathrm{del}$ 25 de enero de 2012 y se toman otras determinaciones. http://portal.anla.gov.co/ gaceta/resolucion-0078-del-28-enero-2016 
Autoridad Nacional de Licencias Ambientales. (2017, 21 de marzo). Resolución 297. Por la cual se otorga una licencia ambiental y se toman otras determinaciones. http:// portal.anla.gov.co/gaceta/resolucion-0297-del-21-marzo-2017

Autoridad Nacional de Licencias Ambientales. (2017, 23 de marzo). Resolución 303. Por la cual se otorga una licencia ambiental y se toman otras determinaciones. http:// portal.anla.gov.co/gaceta/resolucion-0303-del-23-marzo-2017

Autoridad Nacional de Licencias Ambientales. (2017, 8 de septiembre). Resolución 1092. Por la cual se otorga una licencia ambiental. http://portal.anla.gov.co/gaceta/ resolucion-1092-del-8-septiembre-2017

Bajo Sanjuán, A. (2017, abril 25). Economía circular: de la cuna a la cuna. http://entreparentesis.org/economia-circular-la-cuna-la-cuna/

Bauman, Z. (2017). Tiempos líquidos: vivir en una época de incertidumbre. Tusquets.

Cámara de Comercio de Urabá. (2016). Informe socioeconómico 2016. https://ccuraba. org.co/informe/informe-socioeconomico-2016/

Campillo, A. (2015). Tierra de nadie: cómo pensar (en) la sociedad global. Herder.

Congreso de Colombia. (2011, 10 de junio). Ley 1448. Por la cual se dictan medidas de atención, asistencia y reparación integral a las víctimas del conflicto armado interno y se dictan otras disposiciones. Diario Oficial 48096. http://www.suin-juriscol.gov.co/ viewDocument.asp?ruta =Leyes $/ 1680697$

Corte Interamericana de Derechos Humanos. Caso de la masacre de Pueblo Bello vs. Colombia. Sentencia de 31 de enero de 2006. http://www.corteidh.or.cr/docs/casos/articulos/seriec_140_esp.pdf

Escobar, A. (2014). Sentipensar con la tierra. Disponible en: http://biblioteca.clacso.edu. ar/Colombia/escpos-unaula/20170802050253/pdf_460.pdf

Galtung, J. (2003). La violencia cultural. Red Gernika. https://www.gernikagogoratuz. org/portfolio-item/violencia-cultural-galtung/

Guattari, F. (2015). ¿Qué es la ecosofía? (trad. I. de Pablo). Cactus.

Logistica424. (s. f.). Puerto Turbo. https://logistica424.wordpress.com/puerto-turbo/

Mouffe, C. (1999). El retorno de lo político: comunidad, ciudadanía, pluralismo, democracia radical. Paidós.

Naess, A. (s. f.). Biocentrismo: ecología profunda. https://www.uv.mx/orizaba/cosustenta/files/2015/05/Biocentrismo-Ecologia-Profunda.pdf

Noguera, Ramírez, Echeverri. (). Métodoestesis: los caminos del sentir en los saberes de la tierra una aventura geo-epistémica en clave sur. Disponible en:

Métodoestesis | Revista de Investigación Agraria y Ambiental (unad.edu.co)

Sarmiento Ortiz, C. (2007). Urabá: pulsiones de vida y desafíos de muerte. La Carreta.

Serrano Gómez, E. (2001). Filosofía del conflicto político. Fondo de Cultura Económica.

Serres, M. (2004). El contrato natural (trad. J. Vásquez Pérez y U. Larraceleta). Pre-textos. 